\title{
The Roles of Glial Cell Line-Derived Neurotrophic Factor, Tumor Necrosis Factor- $\alpha$, and an Inducer of These Factors in Drug Dependence
}

\author{
Minae Niwa ${ }^{1,2}$, Atsumi Nitta ${ }^{1}$, Kiyofumi Yamada ${ }^{1,3}$, and Toshitaka Nabeshima ${ }^{1,2, *}$ \\ ${ }^{1}$ Department of Neuropsychopharmacology and Hospital Pharmacy, Nagoya University Graduate School of Medicine, \\ Nagoya 466-8560, Japan \\ ${ }^{2}$ Department of Chemical Pharmacology, Meijo University Graduate School of Pharmaceutical Sciences, \\ Nagoya 468-8503, Japan \\ ${ }^{3}$ Laboratory of Neuropsychopharmacology, Division of Life Sciences, Graduate School of Natural Science and Technology, \\ Kanazawa University, Kanazawa 920-1192, Japan
}

Received January 26, 2007; Accepted April 17, 2007

\begin{abstract}
There are few efficacious medications for drug dependence at present. Recent evidence has suggested that various cytokines are involved in the effects of abused drugs, suggesting that these factors play a role in drug dependence. In this article, the roles of glial cell line-derived neurotrophic factor (GDNF) and tumor necrosis factor- $\alpha$ (TNF- $\alpha$ ) in drug dependence are discussed. GDNF inhibits the cocaine-induced upregulation of tyrosine hydroxylase activity in the ventral tegmental area and blocks behavioral responses to cocaine. TNF- $\alpha$ attenuates rewarding effects and locomotor sensitization induced by methamphetamine (METH) and morphine (MOR). Moreover, we mentioned the potential of Leu-Ile, which induces the expression of GDNF and TNF- $\alpha$, as a novel therapeutic agent for drug dependence. Leu-Ile inhibits not only the development but also the maintenance of METH- or MOR-induced place preference and locomotor sensitization in mice. The inhibitory effect of Leu-Ile on METH- or MOR-induced place preference is not observed in GDNF heterozygous and TNF- $\alpha$ knockout mice. Leu-Ile inhibits METH- or MOR-induced place preference and sensitization by attenuating the METH- or MOR-induced increase in extracellular dopamine levels in the nucleus accumbens via the induction of GDNF and TNF- $\alpha$ expression. These findings suggest that Leu-Ile could be a novel therapeutic agent for drug dependence.
\end{abstract}

Keywords: glial cell line-derived neurotrophic factor, tumor necrosis factor- $\alpha$, methamphetamine, morphine, Leu-Ile

\section{Introduction}

Drugs of abuse are able to elicit compulsive drugseeking behaviors upon repeated administration, which ultimately leads to the phenomenon of addiction (1). In terms of lost lives and productivity, drug addiction remains one of the most serious threats to a nation's public health (2). There are currently few efficacious medications for drug dependence. Recently, it has been reported that BP897, a dopamine (DA) $\mathrm{D}_{3}$-receptor

*Corresponding author (affiliation \#2).

tnabeshi@ccmfs.meijo-u.ac.jp

Published online in J-STAGE: May 31, 2007

doi: 10.1254/jphs.CP0070017 partial agonist, affects cocaine-associated stimulusinduced drug-seeking behavior in rats (3). The opioid $\kappa$ receptor agonist TRK-820 inhibits not only the rewarding effects of morphine (MOR) and cocaine, but also a mecamylamine-precipitated nicotine-withdrawal aversive effect (4). These medications should be effective even when they are administered after the development of drugs of abuse.

Cytokines, which are known to influence synaptic transmission and neuronal morphology (5), may be involved in alterations of the morphology of dendrites and their spines in the prefrontal cortex, nucleus accumbens (NAc), and hippocampus after repeated injection of psychostimulants $(6,7)$. Glial cell line- 
derived neurotrophic factor (GDNF) inhibits the cocaine-induced upregulation of tyrosine hydroxylase (TH) activity in the ventral tegmental area (VTA) and blocks behavioral responses to cocaine (8). Furthermore, we have previously demonstrated that tumor necrosis factor- $\alpha$ (TNF- $\alpha$ ), an inflammatory cytokine, attenuates rewarding effects and locomotor sensitization induced by methamphetamine $(\mathrm{METH})$ and $\operatorname{MOR}(9,10)$. Taken together, GDNF and TNF- $\alpha$ would be candidates for therapeutic agents against drug dependence. However, there are serious obstacles to their therapeutic application: it is difficult to deliver GDNF from the periphery to the brain, since it is a macromolecule that cannot penetrate the blood-brain barrier (11), and is easily broken down by proteases in the blood stream. In addition, TNF- $\alpha$ damages the peripheral tissues, because it triggers the expression of other cytokines (12). Therefore, GDNF and TNF- $\alpha$ cannot be used directly as therapeutic tools for drug dependence. We hypothesized that a low-molecular-weight compound, which can easily penetrate the blood-brain barrier and induces production of GDNF and TNF- $\alpha$ in the brain, could be a novel therapeutic agent for drug dependence.

\section{Roles of GDNF in drug dependence}

GDNF produced by a glial cell line is a factor that plays important roles in the dopaminergic neurons (13). GDNF promotes the survival and function of DA neurons in vivo, in both the intact rat brain and after nigrostriatal lesioning $(14,15)$. It has been also shown that GDNF is secreted in the target (striatum) and transported retrogradely to the DA cell bodies in the mesencephalon (16). These findings suggest that GDNF could be effective against dopaminergic degeneration, and is expected to be useful for dopaminergic neurological disorders.

Transplantation of simian virus- 40 glial cells, which produces and secretes GDNF, or delivery of GDNFconjugated nanoparticles into dorsal and ventral striatum impairs the acquisition of cocaine self-administration in rats $(17,18)$. The upregulation of the GDNF pathway in the midbrain, is the molecular mechanism by which the putative anti-addiction drug ibogaine mediates its desirable action of reducing ethanol consumption (19). Infusion of GDNF into the VTA blocks certain biochemical adaptations (induction of TH, NR1 subunit of $N$-methyl D-aspartate receptors, $\triangle \mathrm{FosB}$, and protein kinase A catalytic subunit) to chronic cocaine or MOR treatment as well as cocaine-induced place preference (8). Conditioned place preference (CPP) paradigm, in which animals learn the association of an environment paired with drug exposure, is usually used as a measure of the rewarding properties of abused drugs $(8-10)$. Conversely, responses to cocaine are enhanced in rats by intra-VTA infusion of anti-GDNF antibody and in GDNF heterozygous knockout mice (8). Chronic exposure to cocaine or MOR causes a dramatic decrease in GDNF signaling as evidenced by a $65 \%-70 \%$ reduction in the levels of tyrosine-phosphorylated Ret in the VTA (8). Taken together, these findings support the scheme that the drug-induced decrease in GDNF signaling in the VTA removes a homeostatic feedback (or counteracting) mechanism on this neural pathway and thereby contributes to sensitized biochemical and behavioral responses to drugs of abuse.

\section{Roles of TNF- $\alpha$ in drug dependence}

TNF, a potent proinflammatory cytokine, plays a critical role in the immune response that leads to effective clearance of a variety of pathogens or to immunopathology and autoimmune disease (20). TNF- $\alpha$ also affects the central nervous system (CNS) directly or indirectly through the stimulation of vagal afferents (21). Thus, this cytokine is emerging as a modulator of CNS function. Regarding the behavioral effects of TNF- $\alpha$, transgenic mice expressing high levels of TNF- $\alpha$ in the brain show several changes in exploratory activity and emotional behavior in association with reduced $\mathrm{TH}$ immunoreactivity in the striatum without neuronal cell death (22). In contrast, mice with targeted deletions of the TNF- $\alpha$ gene (TNF- $\alpha$ knockout mice) show anxiogenic-like behavior accompanied by an increase in serotonin metabolism (23).

METH or cocaine induces the expression of TNF- $\alpha$ mRNA in human brain endothelial cells $(24,25)$. METH induces the expression of TNF- $\alpha$ mRNA and protein in the NAc, striatum, hippocampus, and amygdala of rats (9). Expression of the TNF- $\alpha$ in the brain of mice is induced $3 \mathrm{~h}$ after METH injection and remains elevated for up to $6 \mathrm{~h}$ of METH exposure (26). Repeated METH or MOR treatment increases TNF- $\alpha$ mRNA and protein levels in neurons of the NAc through the activation of DA receptors $(9,10)$. Recently, we have demonstrated that TNF- $\alpha$ plays a neuroprotective role in METHinduced dependence and neurotoxicity (9) (Table 1). Moreover, we have found that TNF- $\alpha$ inhibits MORinduced rewarding effects and sensitization to the locomotor-stimulating effects, which is argued to reflect one neuroadaptive process associated with dependence (10) (Table 1). Conversely, responses to METH or MOR are enhanced in TNF- $\alpha$ knockout mice. Furthermore, TNF- $\alpha$ attenuates the repeated METH or MOR treatment-induced increase in extracellular DA levels in the NAc $(9,10)$. TNF- $\alpha$ itself potentiates synaptosomal and 
Table 1. Summary of the effects of TNF- $\alpha$ on methamphetamineor morphine-induced changes in biomarkers and behavior

\begin{tabular}{lcc}
\hline & Methamphetamine & Morphine \\
\hline TNF- $\alpha$ mRNA & & \\
Single & $\uparrow$ & \pm \\
Repeated & $\uparrow$ & $\uparrow$ \\
\hline Conditioned place preference & $\uparrow$ & $\uparrow$ \\
Wild type & $\downarrow$ & $\downarrow$ \\
Wild type + TNF- $\alpha$ & $\uparrow \uparrow$ & $\uparrow \uparrow$ \\
TNF- $\alpha(-/-)$ & N.D. & $\downarrow$ \\
TNF- $\alpha(-/-)+$ TNF- $\alpha$ &
\end{tabular}

Hyperlocomotion (Single)

Wild type

Wild type + TNF- $\alpha$

TNF- $\alpha(-/-)$

$\begin{array}{ll}\uparrow & \uparrow \\ \downarrow & \uparrow \\ \uparrow & \end{array}$

Locomotor sensitization (Repeated)

Wild type

Wild type + TNF- $\alpha$

TNF- $\alpha(-/-)$

$\begin{array}{lll}\uparrow & \uparrow \\ \downarrow & \downarrow \\ \uparrow & \downarrow\end{array}$

Extracellular dopamine levels

Wild type

Wild type + TNF- $\alpha$

$\uparrow \uparrow$

N.D.

\pm , No change; $\uparrow$, Significant increase; $\downarrow$, Significant decrease; N.D., Not determined.

vesicular DA uptake and diminishes the METH-induced decrease in synaptosomal and vesicular DA uptake (9). These findings suggest that TNF- $\alpha$ plays a neuroprotective role in METH- or MOR-induced dependence by activating plasmalemmal DA transporter as well as vesicular monoamine transporter-2 and by inhibiting METH- or MOR-induced DA release $(9,10)$ (Table 1$)$.

\section{Effects of Leu-Ile, an inducer for GDNF and TNF- $\alpha$, on METH-induced dependence}

GDNF and TNF- $\alpha$ inhibit drug-induced dependence and would be candidates for therapeutic agents against drug dependence. Furthermore, a previous study has demonstrated that inflammatory stimuli such as TNF- $\alpha$ and lipopolysaccharide induces the synthesis of GDNF cultured astrocytes from mouse brain (27). However, as described at the beginning of the article, GDNF and TNF- $\alpha$ as anti-addictive factors cannot be used directly as therapeutic tools for drug dependence. Therefore, we hypothesized that a low molecular weight compound that can induce production of GDNF and TNF- $\alpha$ in the brain would be useful as a novel therapeutic agent for drug dependence. We investigated whether certain hydrophobic dipeptides, Leu-Ile, Leu-Pro, and Pro-Ile, that partially resemble the immunophilin-binding site on FK506 have neuroprotective effects both in vivo and in vitro attributable to the induction of GDNF (28). Nitta et al. have demonstrated that only Leu-Ile induces GDNF, but not Leu-Pro and Pro-Ile (28). Leu-Ile upregulates GDNF expression by activating heat shock protein 90 / Akt / cyclic adenosine 3',5'-monophosphase response element binding protein signaling (29). Leu-Ile induces TNF- $\alpha$ mRNA in the NAc (10), although the mechanism underlying the upregulation of TNF- $\alpha$ induced by Leu-Ile remains to be elucidated. Taken together, Leu-Ile is expected to induce the production of not only GDNF but also TNF- $\alpha$ and to inhibit drug dependence.

The levels of GDNF mRNA, GDNF, and TNF- $\alpha$ protein are significantly increased $24 \mathrm{~h}$ after addition of Leu-Ile compared with those in the control group in primary cultured hippocampal neurons (30). In vivo, Leu-Ile treatment not only in combination with METH, but also after withdrawal from repeated treatment with METH increases GDNF and TNF- $\alpha$ levels in the NAc of mice (30). GDNF is expressed in the neuronal and astro glial cells of the NAc after the repeated co-administration of Leu-Ile and METH, whereas TNF- $\alpha$ is expressed in the neurons in mouse brain (30).

Leu-Ile treatment, in combination with METH and after withdrawal from repeated treatment with METH, inhibits place preference and locomotor sensitization to METH. GDNF and TNF- $\alpha$ are involved in the inhibitory effects of Leu-Ile on the rewarding effects of METH because no effects of Leu-Ile are observed in the GDNF heterozygous and TNF- $\alpha$ knockout mice (30).

To explore the mechanisms of the inhibitory effects of Leu-Ile on METH-induced rewarding effects and locomotor sensitization, the effects of Leu-Ile on the increase in extracellular DA levels and the decrease in DA uptake into synaptosomes induced by repeated METH treatment are examined by using in vivo microdialysis and the $\left[{ }^{3} \mathrm{H}\right] \mathrm{DA}$ uptake assay, respectively. Leu-Ile inhibits the repeated METH treatment-induced increase in extracellular DA levels in the NAc and negates the METHinduced inhibition of DA uptake in the midbrain. These findings suggest that Leu-Ile inhibits METH-induced rewarding effects and locomotor sensitization by regulating extracellular DA levels via the induction of GDNF and TNF- $\alpha$ expressions (30) (Table 2).

\section{Effects of Leu-Ile, an inducer for GDNF and TNF- $\alpha$, on MOR-induced dependence}

Leu-Ile potentiates the MOR-induced increase in GDNF levels in the NAc of mice (31). Moreover, 
Table 2. Summary of the effects of Leu-Ile on methamphetamineor morphine-induced changes in biomarkers and behavior

\begin{tabular}{lcc}
\hline & Methamphetamine & Morphine \\
\hline GDNF levels & $\uparrow$ & $\uparrow$ \\
Vehicle & $\uparrow \uparrow$ & $\uparrow \uparrow$ \\
Leu-Ile & & \\
\hline TNF- $\alpha$ levels & $\uparrow$ & $\uparrow$ \\
Vehicle & $\uparrow \uparrow$ & $\uparrow \uparrow$ \\
Leu-Ile & & \\
\hline Conditioned place preference & $\uparrow$ & $\uparrow$ \\
Vehicle & $\downarrow$ & $\downarrow$ \\
Leu-Ile & $\uparrow$ & $\uparrow$ \\
\hline Hyperlocomotion (Single) & $\uparrow$ & $\uparrow$ \\
Vehicle & $\uparrow$ & \\
Leu-Ile & & $\uparrow$ \\
\hline Locomotor sensitization (Repeated) & $\uparrow$ & $\downarrow$ \\
Vehicle & $\downarrow$ & $\uparrow$ \\
Leu-Ile & & $\downarrow$ \\
\hline Extracellular dopamine levels & $\uparrow$ & \\
Vehicle & & \\
Leu-Ile & & \\
\hline
\end{tabular}

$\uparrow$, Significant increase; $\downarrow$, Significant decrease.

Leu-Ile treatment, in combination with MOR and after withdrawal from repeated treatment with MOR, also increases TNF- $\alpha$ levels in the neuronal cells of the NAc (10).

The effects of Leu-Ile on the MOR-induced rewarding effects and locomotor sensitization are examined in the CPP paradigm and locomotor activity test, respectively. Leu-Ile treatment, in combination with MOR and after withdrawal from repeated treatment with MOR, inhibits place preference and locomotor sensitization to MOR. GDNF and TNF- $\alpha$ are involved in the inhibitory effects of Leu-Ile on the rewarding effects of MOR, because no effects of Leu-Ile are observed in the GDNF heterozygous and TNF- $\alpha$ knockout mice $(10,31)$.

To explore the mechanisms of the inhibitory effects of Leu-Ile on MOR-induced rewarding effects and locomotor sensitization, the effects of Leu-Ile on the increase in extracellular DA levels induced by repeated MOR treatment are examined by using in vivo microdialysis. Leu-Ile inhibits the repeated MOR treatment-induced increase in extracellular DA levels in the NAc (10). These findings suggest that Leu-Ile inhibits MORinduced rewarding effects and locomotor sensitization by regulating extracellular DA levels via the induction of GDNF and TNF- $\alpha$ expression $(10,31)$ (Table 2).

\section{Conclusions}

GDNF and TNF- $\alpha$ play important roles in the rewarding effects of both METH and MOR $(8-10)$ (Table 2). Since the deletion of GDNF or TNF- $\alpha$ genes results in an enhancement of the rewarding effects, these cytokines may reduce the rewards of METH and MOR $(8-10)$ (Table 1). As reviewed in this article, some cytokines like GDNF and TNF- $\alpha$ act as anti-addictive factors, whereas others such as tissue plasminogen activator (tPA), metalloproteinase (MMP)-2, MMP-9, basic fibroblast growth factor, neurotropin-3, and brainderived neurotrophic factor (BDNF) act as pro-addictive factors $(32-34)$. The tPA-plasmin system, for instance, potentiates the rewarding and locomotor-stimulating effects of METH, MOR, and nicotine by regulating release of DA (34-36). MMP-2 or -9-deficient mice show reduced responses to METH in locomotor sensitization, CPP, and dopamine release in the NAc (33). Infusion of BDNF into the NAc enhances the stimulation of locomotor activity by cocaine in rats, whereas the development of sensitization and CPP is delayed in heterozygous BDNF knockout mice compared with their wild-type littermates $(37,38)$. The balance of levels between anti-addictive and pro-addictive factors induced by drugs of abuse seems to be important to the development of drug dependence (32). We propose that the dynamic changes and balance of levels in antiaddictive and pro-addictive factors in the brain are some of the determinants of the susceptibility to drug dependence.

As described at the beginning of the article, GDNF and TNF- $\alpha$ as anti-addictive factors cannot be used directly as therapeutic tools for drug dependence. In this article, we demonstrated that Leu-Ile, a low molecular weight compound that induces production of GDNF and TNF- $\alpha$ in the brain, inhibits both METH- and MORinduced rewarding effects and locomotor sensitization by regulating extracellular DA levels via the induction of GDNF and TNF- $\alpha$ expression $(10,30,31)$ (Table 2). Therefore, Leu-Ile could be a novel therapeutic agent for METH- and MOR-induced dependence.

\section{Acknowledgments}

This study was supported in part by a Grant-in-Aid for Scientific Research and Special Coordination Funds for Promoting Science and Technology, Target-Oriented Brain Science Research Program; by a Grant-in-aid for Scientific Research (B) and Young Scientists (A); by the 21st Century Center of Excellence Program "Integrated Molecular Medicine for Neuronal and Neoplastic Disorders" from the Ministry of Education, Culture, 
Sports, Science, and Technology of Japan; by a Grantin-Aid for Health Science Research on Regulatory Science of Pharmaceuticals and Medical Devices, and Comprehensive Research on Aging and Health from the Ministry of Health, Labor, and Welfare of Japan; by a Smoking Research Foundation Grant for Biomedical Research; by the Mochida Memorial Foundation for Medical and Pharmaceutical Research; by a grant from the Brain Research Center from the 21st Century Frontier Research Program funded by the Ministry of Science and Technology, Republic of Korea; and by Japan Canada Joint Health Research Program.

\section{References}

1 Laakso A, Mohn AR, Gainetdinov RR, Caron MG. Experimental genetic approaches to addiction. Neuron. 2002;36:213228.

2 Nestler EJ. From neurobiology to treatment: progress against addiction. Nat Neurosci. 2002;5:1076-1079.

3 Cervo L, Carnovali F, Stark JA, Mennini T. Cocaine-seeking behavior in response to drug-associated stimuli in rats: involvement of D3 and D2 dopamine receptors. Neuropsychopharmacology. 2003;28:1150-1159.

4 Hasebe K, Kawai K, Suzuki T, Kawamura K, Tanaka T, Narita $\mathrm{M}$, et al. Possible pharmacotherapy of the opioid $\kappa$ receptor agonist for drug dependence. Ann N Y Acad Sci. 2004;1025: 404-413.

5 Neumann H, Schweigreiter R, Yamashita T, Rosenkranz K, Wekerle H, Barde Y. Tumor necrosis factor inhibits neurite outgrowth and branching of hippocampal neurons by a Rhodependent mechanism. J Neurosci. 2002;22:854-862.

6 Williams MT, Brown RW, Vorhees CV. Neonatal methamphetamine administration induces region-specific long-term neuronal morphological changes in the rat hippocampus, nucleus accumbens and parietal cortex. Eur J Neurosci. 2004;191:3165-3170.

7 Robinson TE, Kolb B. Alterations in the morphology of dendrites and dendritic spines in the nucleus accumbens and prefrontal cortex following repeated treatment with amphetamine or cocaine. Eur J Neurosci. 1999;11:1598-1604.

8 Messer CJ, Eisch AJ, Carlezon WA Jr, Whisler K, Shen L, Wolf $\mathrm{DH}$, et al. Role for GDNF in biochemical and behavioral adaptations to drugs of abuse. Neuron. 2000;26:247-257.

9 Nakajima A, Yamada K, Nagai T, Uchiyama T, Miyamoto Y, Mamiya T, et al. Role of tumor necrosis factor- $\alpha$ in methamphetamine-induced drug dependence and neurotoxicity. J Neurosci. 2004;24:2212-2225.

10 Niwa M, Nitta A, Yamada Y, Nakajima A, Saito K, Seishima M, et al. Tumor necrosis factor- $\alpha$ and its inducer inhibit morphineinduced rewarding effects and sensitization. Biol Psychiatry. In press 2007.

11 Lin LF, Doherty DH, Lile JD, Bektesh S, Collins F. GDNF: a glial cell line-derived neurotrophic factor for midbrain dopaminergic neurons. Science. 1993;260:1130-1132.

12 Bluthe RM, Pawlowski M, Suarez S, Parnet P, Pittman Q, Kelley KW, et al. Synergy between tumor necrosis factor $\alpha$ and interleukin-1 in the induction of sickness behavior in mice. Psychoneuroendocrinology. 1994;19:197-207.
13 Schubert D, Heinemann S, Carlisle W, Tarikas H, Kimes B, Patrick J, et al. Clonal cell lines from the rat central nervous system. Nature. 1974;249:224-227.

14 Bowenkamp KE, Hoffman AF, Gerhardt GA, Henry MA, Biddle PT, Hoffer BJ, et al. Glial cell line-derived neurotrophic factor supports survival of injured midbrain dopaminergic neurons. J Comp Neurol. 1995;355:479-489.

15 Tomac A, Lindqvist E, Lin LF, Ogren SO, Young D, Hoffer BJ, et al. Protection and repair of the nigrostriatal dopaminergic system by GDNF in vivo. Nature. 1995;373:335-339.

16 Tomac A, Widenfalk J, Lin LF, Kohno T, Ebendal T, Hoffer BJ, et al. Retrograde axonal transport of glial cell line-derived neurotrophic factor in the adult nigrostriatal system suggests a trophic role in the adult. Proc Natl Acad Sci U S A. 1995; 92:8274-8278.

17 Green-Sadan T, Kinor N, Roth-Deri I, Geffen-Aricha R, Schindler CJ, Yadid G. Transplantation of glial cell line-derived neurotrophic factor-expressing cells into the striatum and nucleus accumbens attenuates acquisition of cocaine selfadministration in rats. Eur J Neurosci. 2003;18:2093-2098.

18 Green-Sadan T, Kuttner Y, Lublin-Tennenbaum T, Kinor N, Boguslavsky Y, Margel S, et al. Glial cell line-derived neurotrophic factor-conjugated nanoparticles suppress acquisition of cocaine self-administration in rats. Exp Neurol. 2005;194:97105.

19 He DY, McGough NN, Ravindranathan A, Jeanblanc J, Logrip ML, Phamluong K, et al. Glial cell line-derived neurotrophic factor mediates the desirable actions of the anti-addiction drug ibogaine against alcohol consumption. J Neurosci. 2005;25:619628.

20 Vassali P. The pathophysiology of tumor necrosis factors. Annu Rev Immunol. 1992;10:411-452.

21 Maier SF, Watkins LR. Cytokines for psychologists: implications of bidirectional immune-to-brain communication for understanding behavior, mood, and cognition. Psychol Rev. 1998;105:83-107.

22 Aloe L, Fiore M. TNF- $\alpha$ expressed in the brain of transgenic mice lowers central tyrosine hydroxylase immunoreactivity and alters grooming behavior. Neurosci Lett. 1997;238:65-68.

23 Yamada K, Iida R, Miyamoto Y, Saito K, Sekikawa K, Seishima $\mathrm{M}$, et al. Neurobehavioral alterations in mice with a targeted deletion of the tumor necrosis factor- $\alpha$ gene: implications for emotional behavior. J Neuroimmunol. 2000;111:131-138.

24 Lee YW, Hennig B, Yao J, Toborek M. Methamphetamine induces AP-1 and NF- $\kappa \mathrm{B}$ binding and transactivation in human brain endothelial cells. J Neurosci Res. 2001;66:583-591.

25 Lee YW, Hennig B, Fiala M, Kim KS, Toborek M. Cocaine activates redox-regulated transcription factors and induces TNF- $\alpha$ expression in human brain endothelial cells. Brain Res. 2001;920:125-133.

26 Flora G, Lee YW, Nath A, Maragos W, Henning B, Toborek M. Methamphetamine-induced TNF- $\alpha$ gene expression and activation of AP-1 in discrete regions of mouse brain. Neuromolecular Med. 2002;2:71-85.

27 Appel E, Kolman O, Kazimirsky G, Blumberg PM, Brodie C. Regulation of GDNF expression in cultured astrocytes by inflammatory stimuli. Neuroreport. 1997;8:3309-3312.

28 Nitta A, Nishioka H, Fukumitsu H, Furukawa Y, Sugiura H, Shen L, et al. Hydrophobic dipeptide Leu-Ile protects against neuronal death by inducing brain-derived neurotrophic factor 
and glial cell line-derived neurotrophic factor synthesis. J Neurosci Res. 2004;78:250-258.

29 Cen X, Nitta A, Ohya S, Zhao Y, Ozawa N, Mouri A, et al. An analog of a dipeptide-like structure of FK506 increases glial cell line-derived neurotrophic factor expression through cAMP response element-binding protein activated by heat shock protein 90/Akt signaling pathway. J Neurosci. 2006;26:33353344.

30 Niwa M, Nitta A, Yamada Y, Nakajima A, Saito K, Seishima M, et al. An inducer for glial cell line-derived neurotrophic factor and tumor necrosis factor- $\alpha$ protects methamphetamine-induced rewarding effects and sensitization. Biol Psychiatry. 2007;61: 890-901.

31 Niwa M Nitta A, Shen L, Noda Y, Nabeshima T. Involvement of glial cell line-derived neurotrophic factor in inhibitory effects of a hydrophobic dipeptide Leu-Ile on morphine-induced sensitization and rewarding effects. Behav Brain Res. 2007;179:167-171.

32 Yamada K, Nabeshima T. Pro- and anti-addictive neurotrophic factors and cytokines in psychostimulant addiction: mini review. Ann N Y Acad Sci. 2004;1025:198-204.

33 Mizoguchi H, Yamada K, Niwa M, Mouri A, Mizuno T, Noda $\mathrm{Y}$, et al. Reduction of methamphetamine-induced sensitization and reward in matrix metalloproteinase-2 and -9-deficient mice.
J Neurochem. 2007;100:1579-1588.

34 Nagai T, Yamada K, Yoshimura M, Ishikawa K, Miyamoto Y, Hashimoto K, et al. The tissue plasminogen activator-plasmin system participates in the rewarding effect of morphine by regulating dopamine release. Proc Natl Acad Sci U S A. 2004; 101:3650-3655.

35 Nagai T, Noda Y, Ishikawa K, Miyamoto Y, Yoshimura M, Ito $\mathrm{M}$, et al. The role of tissue plasminogen activator in methamphetamine-related reward and sensitization. J Neurochem. 2005;92:660-667.

36 Nagai $T$, Ito $M$, Nakamichi $N$, Mizoguchi $H$, Kamei $H$, Fukakusa A, et al. The rewards of nicotine: regulation by tissue plasminogen activator-plasmin system through protease activated receptor-1. J Neurosci. 2006;26:12374-12383.

37 Horger BA, Iyasere CA, Berhow MT, Messer CJ, Nestler EJ, Taylor JR. Enhancement of locomotor activity and conditioned reward to cocaine by brain-derived neurotropic factor. J Neurosci. 1999;19:4110-4122.

38 Hall FS, Drgonova J, Goeb M, Uhl GR. Reduced behavioral effects of cocaine in heterozygous brain-derived neurotropic factor (BDNF) knockout mice. Neuropsychopharmacology. 2003;28:1485-1490. 